\title{
Optimal Control of Time Delay Systems via Hybrid of Block-Pulse Functions and Orthonormal Taylor Series
}

\author{
M. Dadkhah ${ }^{1}$ (D) - M. H. Farahi ${ }^{2}$
}

Published online: 17 April 2015

(C) Springer India Pvt. Ltd. 2015

\begin{abstract}
A new method to find the optimal control of time delay systems with quadratic performance index is discussed. The method is based on hybrid functions. The properties of the hybrid functions which consists of block-pulse functions and orthonormal Taylor series are presented. The operational matrices of integration, delay, dual and product are used to reduce the solution of optimal control time delay system to the solution of algebraic equations. Numerical examples are included to illustrate the effectiveness and validity of the technique.
\end{abstract}

Keywords Time delay system · Orthonormalization - Operational matrices · Hybrid functions

\section{Introduction}

The dynamics of many control systems may be expressed by time-delay equations. The delay(s) may appear in the system state, control input and/or output. Delays occur frequently in incubation periods, mechanics, viscoelasticity, physics, physiology, population dynamics, communication, information technologies, stability of networked control systems, maturation times, age structure, blood transfusions, biological, chemical, electronic and transportation systems [1-3]. Therefore the control of time-delay systems has been interested by many engineers and scientists, due to its variety presence in realistic models of phenomena. Since the analytical methods, especially in optimal control of time-delay systems, have less implementation ability and the application of Pontryagin's maximum principle to the optimization of control systems with time-delays as outlined by Kharatishvili [4] results in a system of

$\bowtie \quad$ M. Dadkhah

m_dadkhah@pnu.ac.ir

M. H. Farahi

farahi@math.um.ac.ir

1 Payamenoor University, Tehran, Iran

2 Ferdowsi University of Mashhad, Mashhad, Iran 
coupled two-point boundary-value (TPBV) problem involving both delay and advance terms whose exact solution, except in very special cases, is very difficult [5], different numerical methods have been devised to overcome the problems arising from the application of analytical methods. In other word, the main object of all computational aspects of optimal time-delay systems have been to produce a new method to avoid the solution of the mentioned (TPBV) problem. Orthogonal functions (OFs) and polynomial series have received considerable attentions in dealing with various problems of dynamic systems [6]. For such kind of problem, the approach is that of converting the underlaying differential equations govering the dynamical system to an algebraic form through the use of an operational matrix of integration which can be uniquely determined based on the particular OFs. Special attentions have been given to applications of Walsh functions [7], block-pulse functions [8-10], Laguerre polynomials [11], Legendre polynomials [12-14], Chebyshev polynomials [15,16], Taylor series [17,18] and Fourier series [19,20].

The aim of present paper is to introduce a new numerical method to solve the quadratic optimal control problem with delay systems. This method consists of reducing the optimal control problem to a set of algebraic equations by expanding the state and control vectors as hybrid functions with unknown coefficients. These hybrid functions, which consist of block-pulse functions and orthonormal Taylor series are given. The operational matrices of integration and delay are introduced. The necessary conditions of optimality are derived as a system of algebraic equations in the unknown coefficients of state and control vectors and Lagrange multipliers. These coefficients are determined in such a way that the necessary conditions for extremization are imposed. In this paper, we show a novel strategy by using hybrid functions to find the approximate solutions of time delay optimal control problems. In this method, we divided the time interval into $N$ subintervals and approximate the trajectory and control functions by hybrid of block-pulse functions and orthonormal Taylor series. Indeed in applying the method, by increasing the accuracy of the approximate solutions, but the CPU time and computer needed memory reduce nevertheless, since the operational matrices have large number of zero elements and they are mostly sparce.

\section{Hybrid Functions and Their Properties}

Hybrid functions $H_{n, m}(t), n=1,2, \ldots, N, m=0,1, \ldots M-1$; have three arguments; $n$ and $m$ are the order of block-pulse functions and orthonormal Taylor series, respectively, and $\mathrm{t}$ is the normalized time. They are defined on the interval $[0,1)$ as follows (since any interval $[a, b)$ can be shifted to $[0,1)$ therefore we consider $[0,1)$ here)

$$
H_{n, m}(t)= \begin{cases}\sqrt{N} O T_{m}(N t-n+1), & \left(\frac{n-1}{N}\right) \leq t<\frac{n}{N}, \\ 0, & \text { otherwise, }\end{cases}
$$

where $O T_{m}(t)$ 's are orthonormal Taylor series govered by the Gram-Schmidt orthonormalization process on $T_{m}=\left\{1, t, t^{2}, t^{3}, \ldots, t^{m}\right\}$ and the time interval is $[0,1]$ with the weight function $w(t)=1$. For example we have

$$
\begin{aligned}
& O T_{0}(t)=1 \\
& O T_{1}(t)=(2 t-1) \sqrt{3} \\
& O T_{2}(t)=\left(6 t^{2}-6 t+1\right) \sqrt{5} \\
& O T_{3}(t)=(2 t-1)\left(10 t^{2}-10 t+1\right) \sqrt{7}, \\
& O T_{4}(t)=\left(70 t^{4}-140 t^{3}+90 t^{2}-20 t+1\right) \sqrt{9} .
\end{aligned}
$$


A function $f(t)$ belongs to the space $L^{2}[0,1]$ may be expanded by hybrid functions as follows:

$$
f(t)=\sum_{n=1}^{\infty} \sum_{m=0}^{\infty} c_{n, m} H_{n, m}(t) .
$$

By truncating the series (2), we can abtain an approximation for $f(t)$ as follows:

$$
f(t) \simeq \sum_{n=1}^{N} \sum_{m=0}^{M-1} c_{n, m} H_{n, m}(t)=C^{T} H(t)
$$

where

$$
C=\left[c_{1,0} c_{1,1} \ldots c_{1, M-1} c_{2,0} c_{2,1} \ldots c_{2, M-1} \ldots c_{N, 0} \ldots c_{N, M-1}\right]^{T}
$$

and

$$
\begin{array}{r}
H(t)=\left[H_{1,0}(t) H_{1,1}(t) \ldots H_{1, M-1}(t) H_{2,0}(t) H_{2,1}(t) \ldots H_{2, M-1}(t) \ldots\right. \\
\left.H_{N, 0}(t) H_{N, 1}(t) \ldots H_{N, M-1}(t)\right]^{T},
\end{array}
$$

where, $c_{n, m}, n=1,2, \ldots, N, m=0,1, \ldots, M-1$, are the coefficients expansion of the function $f(t)$ in the $n$-th subinterval $\left[\frac{(n-1)}{N}, \frac{n}{N}\right)$.

We have $c_{n, m}=\left\langle f(t) . H_{n, m}(t)\right\rangle$ and $\langle$.$\rangle is the standard inner product on L^{2}[0,1)$.

\section{Operational Matrix of Integration}

We can approximate the integration of $H(t)$ defined in (4) as follows:

$$
\int_{0}^{t} H(s) d s \simeq P_{h} H(t),
$$

where $P_{h}$ is $M N \times M N$ operational matrix for integration and is given as:

$$
P_{h}=\left(\begin{array}{ccccc}
A_{1} & B_{1} & B_{1} & \ldots & B_{1} \\
0 & A_{1} & B_{1} & \ldots & B_{1} \\
0 & 0 & A_{1} & \ldots & B_{1} \\
\vdots & \vdots & \vdots & \ldots & \vdots \\
0 & 0 & 0 & \ldots & A_{1}
\end{array}\right)
$$

where

$$
B_{1}=\frac{1}{N}\left(\begin{array}{ccccc}
1 & 0 & 0 & \ldots & 0 \\
0 & 0 & 0 & \ldots & 0 \\
0 & 0 & 0 & \ldots & 0 \\
\vdots & \vdots & \vdots & \ldots & \vdots \\
0 & 0 & 0 & \ldots & 0
\end{array}\right)_{(M \times M)}
$$


and

$$
A_{1}=\frac{1}{2 N}\left(\begin{array}{ccccccc}
1 & \frac{\sqrt{1} \sqrt{3}}{1 \times 3} & 0 & 0 & 0 & \ldots & 0 \\
-\frac{\sqrt{1} \sqrt{3}}{1 \times 3} & 0 & \frac{\sqrt{3} \sqrt{5}}{3 \times 5} & 0 & 0 & \ldots & 0 \\
0 & -\frac{\sqrt{3} \sqrt{5}}{3 \times 5} & 0 & \frac{\sqrt{5} \sqrt{7}}{5 \times 7} & 0 & \ldots & 0 \\
\vdots & \vdots & \vdots & \ldots & \vdots & \ldots & 0 \\
0 & 0 & 0 & \ldots & \vdots & \ldots & \frac{\sqrt{2 M-1} \sqrt{2 M-3}}{(2 M-1) \times(2 M-3)} \\
0 & 0 & 0 & \ldots & \vdots-\frac{\sqrt{2 M-1} \sqrt{2 M-3}}{(2 M-1) \times(2 M-3)} & 0
\end{array}\right)_{(M \times M)} .
$$

\section{Dual and Product Operational Matrices}

Since $H_{n, m}(t)$ 's are disjoint and orthonormal sets on [0,1), so the dual operational matrix of $H(t)$ is

$$
L=\int_{0}^{1} H(t) H^{T}(t) d t=\mathbf{I},
$$

where $\mathbf{I}$ is $M N$ identity matrix. Also the following property of the product of two hybrid function vectors will be used. Let

$$
H(t) H^{T}(t) C \simeq \tilde{C} H(t),
$$

where $\tilde{C}$ is a $M N \times M N$ product operational matrix. To show the calculation procedure we may choose $N=4$ and $M=3$. Thus we have

$$
\begin{aligned}
C & =\left[c_{1,0} c_{1,1} c_{1,2} \ldots c_{4,0} c_{4,1} c_{4,2}\right]^{T}, \\
H(t) & =\left[H_{1,0}(t) H_{1,1}(t) H_{1,2}(t) \ldots H_{4,0}(t) H_{4,1}(t) H_{4,2}(t)\right]^{T},
\end{aligned}
$$

where

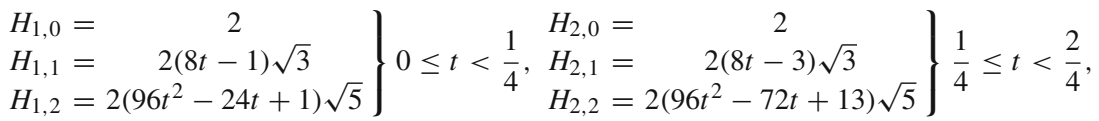

and

$$
\left.\left.\begin{array}{l}
H_{3,0}= \\
H_{3,1}= \\
H_{3,2}=2\left(96 t^{2}-120 t+37\right) \sqrt{5}
\end{array}\right\} \begin{array}{l}
\frac{2}{4} \leq t<\frac{3}{4}, \begin{array}{l}
H_{4,0} \\
H_{4,1}=
\end{array} \quad 2(8 t-7) \sqrt{3} \\
H_{4,2}=2\left(96 t^{2}-168 t+73\right) \sqrt{5}
\end{array}\right\} \frac{3}{4} \leq t<1 .
$$

For example from (1) one can obtain

$$
\begin{aligned}
& H_{1,1}(t)=\sqrt{4} O T_{1}(4 t)=2(8 t-1) \sqrt{3}, \quad 0 \leq t<\frac{1}{4} \\
& H_{2,1}(t)=\sqrt{4} O T_{1}(4 t-1)=2(8 t-3) \sqrt{3}, \quad \frac{1}{4} \leq t<\frac{2}{4} \\
& H_{3,1}(t)=\sqrt{4} O T_{2}(4 t-3)=2(8 t-5) \sqrt{3}, \quad \frac{2}{4} \leq t<\frac{3}{4} \\
& H_{4,1}(t)=\sqrt{4} O T_{2}(4 t-5)=2(8 t-7) \sqrt{3}, \quad \frac{3}{4} \leq t<1,
\end{aligned}
$$


We also have

$$
\begin{aligned}
& H(t) H^{T}(t)
\end{aligned}
$$

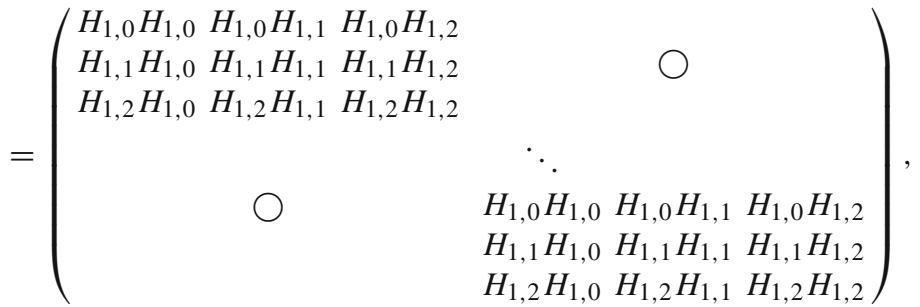

where $\bigcirc$ denoted zero matrix. By using the vector $C$ in (8) the $12 \times 12$ matrix $\tilde{C}$ in (7) is

$$
\tilde{C}=\left(\begin{array}{cccc}
\tilde{C}_{1} & 0 & 0 & 0 \\
0 & \tilde{C}_{2} & 0 & 0 \\
0 & 0 & \tilde{C}_{3} & 0 \\
0 & 0 & 0 & \tilde{C}_{4}
\end{array}\right)
$$

where $\tilde{C}_{i}$ 's, $i=1,2,3,4$ are $3 \times 3$ matrices given by

$$
\tilde{C}_{i}=\left(\begin{array}{ccc}
2 c_{i, 0} & 2 c_{i, 1} & 2 c_{i, 2} \\
2 c_{i, 1} & 2 c_{i, 0}+4 \frac{\sqrt{5}}{5} c_{i, 2} & 4 \frac{\sqrt{5}}{5} c_{i, 1} \\
2 c_{i, 2} & 4 \frac{\sqrt{5}}{5} c_{i, 1} & 2 c_{i, 0}+4 \frac{\sqrt{5}}{7} c_{i, 2}
\end{array}\right) .
$$

\section{Delay Operational Matrix}

The delay function $H(t-\tau)$ is the shifted of the function $H(t)$ defined in (4), along the time axis by $\tau$. In other word we have

$$
H(t-\tau)=D_{\tau} H(t), \quad t>\tau, \quad 0 \leq t \leq 1,
$$

where $D_{\tau}$ is the delay operational matrix of hybrid functions. To find $D_{\tau}$, we first choose $N$ the order of block-pulse functions, as the following manner [5]:

$$
N= \begin{cases}\frac{1}{\tau}, & \frac{1}{\tau} \in \mathbb{Z}, \\ {\left[\frac{1}{\tau}\right]+1} & \text { otherwise, }\end{cases}
$$

where [.] denotes greatest integer value.

Note that in the interval $\tau \leq t \leq 2 \tau$, the terms $H_{1, m}(t)$ for $m=0,1, \ldots, M-1$ are nonzero and all other terms are zero. So if we expand $H_{1, m}(t)$ in terms of $H_{2, m}(t)$ then the coefficients form an $M \times M$ identity matix since we have $H_{1, m}(t-\tau)=H_{2, m}(t)$. Similar manner can be used to all other intervals. Thus if we expand $H(t-\tau)$ in terms of $H(t)$ we find $N M \times N M$ matrix $D_{\tau}$ as

$$
D_{\tau}=\left(\begin{array}{cccccc}
0 & I & 0 & 0 & \ldots & 0 \\
0 & 0 & I & 0 & \ldots & 0 \\
0 & 0 & 0 & I & \ldots & 0 \\
\vdots & \vdots & \vdots & \ldots & \vdots & \\
0 & 0 & 0 & 0 & \ldots & I \\
0 & 0 & 0 & 0 & \ldots & 0
\end{array}\right)
$$

where $I$ is $M \times M$ identity matrix. 


\section{Problem Statement}

Consider the following quadratic time-independent delay control system:

$$
\begin{aligned}
\min \quad J & =\frac{1}{2} x^{T}(1) F x(1)+\frac{1}{2} \int_{0}^{1}\left\{x^{T}(t) S x(t)+u^{T}(t) W u(t)\right\} d t, \\
\text { s.t } \dot{x}(t) & =A x(t)+B x\left(t-\tau_{1}\right)+E u(t)+D u\left(t-\tau_{2}\right), \quad 0 \leq t \leq 1, \\
x(0) & =x_{0}, \\
x(t) & =\theta(t), \quad-\tau_{1} \leq t<0, \\
u(t) & =\psi(t), \quad-\tau_{2} \leq t<0,
\end{aligned}
$$

where $W$ is symmetric positive definite and $F, S$ are positive semi-definite matrices [21], $x(t) \in \mathbb{R}^{p}, u(t) \in \mathbb{R}^{q}$ are state and control vectores respectively and $A, B, E, D$ are matrices of appropriate dimensions, $x_{0}$ is a constant specified vector, and $\theta(t), \psi(t)$ are arbitrary known functions. We choose $W$ as a symmetric positive definite and $F, S$ as positive semi-definite to show that the cost functional $J$ is quadratic and convex, so the necessary conditions for existence of solution, is also sufficient. The problem is to find $x(t)$ and $u(t), 0 \leq t \leq 1$, satisfying (18)-(21) while minimizing (17).

Assume that

$$
\begin{aligned}
x(t) & =\left[x_{1}(t) x_{2}(t) \ldots x_{p}(t)\right]^{T}, \\
u(t) & =\left[u_{1}(t) u_{2}(t) \ldots u_{q}(t)\right]^{T}, \\
\hat{H}(t) & =I_{p} \otimes H(t), \\
\hat{H}_{1}(t) & =I_{q} \otimes H(t),
\end{aligned}
$$

where $I_{p}$ and $I_{q}$ are the $p$ and $q$ dimensional identity matrices and $\otimes$ denotes Kronecker product [22]. Here

$$
\begin{gathered}
\hat{H}(t)=I_{p} \otimes H(t)=\left(\begin{array}{ccc}
H(t) & \ldots & H(t) \\
\vdots & \vdots & \vdots \\
H(t) & \ldots & H(t)
\end{array}\right)_{p \times p}, \\
\hat{H}_{1}(t)=I_{q} \otimes H(t)=\left(\begin{array}{ccc}
H(t) & \ldots & H(t) \\
\vdots & \vdots & \vdots \\
H(t) & \ldots & H(t)
\end{array}\right)_{q \times q} .
\end{gathered}
$$

$\hat{H}(t)$ and $\hat{H}_{1}(t)$ are $p M N \times p$ and $q M N \times q$ matrices respectively while $H(t)$ is the vector function defined in (4). Assume that each of $x_{i}(t)$ and each of $u_{j}(t), i=1,2, \ldots, p, j=$ $1,2, \ldots, q$, can be written in terms of hybrid functions as

$$
\begin{aligned}
x_{i}(t) & =H^{T}(t) X_{i}, \\
u_{j}(t) & =H^{T}(t) U_{j} .
\end{aligned}
$$

Using Eqs. (22)-(25) we have

$$
\begin{aligned}
& x(t)=\hat{H}^{T}(t) X, \\
& u(t)=\hat{H}_{1}^{T}(t) U,
\end{aligned}
$$


where

$$
\begin{aligned}
& X=\left[X_{1} X_{2} \ldots X_{p}\right]^{T}, \\
& U=\left[U_{1} U_{2} \ldots U_{q}\right]^{T} \text {. }
\end{aligned}
$$

Similarly we have

$$
\begin{aligned}
x(0) & =\hat{H}^{T}(t) G, \\
\theta\left(t-\tau_{1}\right) & =\hat{H}^{T}(t) K, \\
\psi\left(t-\tau_{2}\right) & =\hat{H}^{T}(t) R,
\end{aligned}
$$

where

$$
\begin{aligned}
G & =\left[g_{1} g_{2} \ldots g_{p}\right]^{T}, \\
K & =\left[k_{1} k_{2} \ldots k_{p}\right]^{T}, \\
R & =\left[r_{1} r_{2} \ldots r_{q}\right]^{T} .
\end{aligned}
$$

We can also write $x\left(t-\tau_{1}\right)$ and $u\left(t-\tau_{2}\right)$ in terms of hybrid functions as

$$
\begin{aligned}
& x\left(t-\tau_{1}\right)= \begin{cases}\hat{H}^{T}(t) K, & 0 \leq t \leq \tau_{1}, \\
\hat{H}^{T}(t) \hat{D}_{1}^{T} X, & \tau_{1} \leq t \leq 1,\end{cases} \\
& u\left(t-\tau_{2}\right)= \begin{cases}\hat{H}^{T}(t) R, & 0 \leq t \leq \tau_{2}, \\
\hat{H}^{T}(t) \hat{D}_{2}^{T} U, & \tau_{2} \leq t \leq 1,\end{cases}
\end{aligned}
$$

where $\hat{D}_{1}=I_{p} \otimes D_{\tau_{1}}$ and $\hat{D}_{2}=I_{q} \otimes D_{\tau_{2}}$ and $D_{\tau_{1}}, D_{\tau_{2}}$ are respectively delay operational matrices given in (16).

Moreover we have

$$
\begin{aligned}
\int_{0}^{t} \hat{H}^{T}(s) d s & =\left(I_{p} \otimes H^{T}(t)\right)\left(I_{p} \otimes P_{h}^{T}\right)=\hat{H}(t) \hat{P}_{h}^{T}, \\
\int_{0}^{t} x\left(s-\tau_{1}\right) d s & = \begin{cases}\hat{H}^{T}(t) \hat{P}_{h}^{T} K, & 0 \leq t \leq \tau_{1}, \\
\hat{H}^{T}(t) Z_{1} K+\hat{H}^{T}(t) \hat{P}_{h}^{T} \hat{D}_{1}^{T} X, & \tau_{1} \leq t \leq 1,\end{cases} \\
\int_{0}^{t} u\left(s-\tau_{2}\right) d s & = \begin{cases}\hat{H}^{T}(t) \hat{P}_{h}^{T} R, & 0 \leq t \leq \tau_{2}, \\
\hat{H}^{T}(t) Z_{2} R+\hat{H}^{T}(t) \hat{P}_{h}^{T} \hat{D}_{2}^{T} U, & \tau_{2} \leq t \leq 1,\end{cases}
\end{aligned}
$$

where $P_{h}$ is the operational matrix of integration given in (6) and the constant matrices $Z_{1}, Z_{2}$ are

$$
\int_{0}^{\tau_{i}} \hat{H}^{T}(t) d t=\hat{H}^{T}(t) Z_{i}, \quad i=1,2 .
$$

By integrating (18) from 0 to $t$ and using (26)-(33) we have

$$
\begin{aligned}
\hat{H}^{T}(t) X-\hat{H}^{T}(t) G= & A \hat{H}^{T}(t) \hat{P}_{h}^{T} X+B \hat{H}^{T}(t) \hat{P}_{h}^{T} K \\
& +B \hat{H}^{T}(t) Z_{1} K+B \hat{H}^{T}(t) \hat{P}_{h}^{T} \hat{D}_{1}^{T} X \\
& +E \hat{H}^{T}(t) \hat{P}_{h}^{T} U+D \hat{H}^{T}(t) \hat{P}_{h}^{T} R \\
& +D \hat{H}^{T}(t) Z_{2} R+D \hat{H}^{T}(t) \hat{P}_{h}^{T} \hat{D}_{2}^{T} U .
\end{aligned}
$$

From Eq. (34) and by deleting $\hat{H}^{T}(t)$ from both sides we have

$$
\begin{aligned}
C^{*}= & X-G-A \hat{P}_{h}^{T} X-B \hat{P}_{h}^{T} K-B Z_{1} K-B \hat{P}_{h}^{T} \hat{D}_{1}^{T} X \\
& -E \hat{P}_{h}^{T} U-D \hat{P}_{h}^{T} R-D Z_{2} R-D \hat{P}_{h}^{T} \hat{D}_{2}^{T} U=0 .
\end{aligned}
$$


Similarly for J in (17), we have

$$
\begin{aligned}
J(X, U)= & \frac{1}{2} X^{T}\left(H(1) H^{T}(1) \otimes F\right) X+\frac{1}{2} X^{T}(L \otimes S) X \\
& +\frac{1}{2} U^{T}(L \otimes W) U,
\end{aligned}
$$

where $L=\int_{0}^{1} H(t) H^{T}(t) d t$ and indeed we know that $L=I$, where $I$ is $M N$ identity matrix.

The delay optimal control problem has now been reduced to a parameter optimization problem which can be stated as follows. Find $X$ and $U$ so that $J(X, U)$ is minimized subject to the constraints in Eq. (35).

Let

$$
J^{*}(X, U, \lambda)=J(X, U)+\lambda^{T} C^{*},
$$

where the vector $\lambda$ represents the unknown Lagrange multipliers, then the necessary conditions for stationary are given by

$$
\begin{aligned}
& \frac{\partial}{\partial X} J^{*}(X, U, \lambda)=0, \\
& \frac{\partial}{\partial U} J^{*}(X, U, \lambda)=0, \\
& \frac{\partial}{\partial \lambda} J^{*}(X, U, \lambda)=0 .
\end{aligned}
$$

\section{Illustrative Examples}

In this section numerical examples are given to demonstrate the applicability, efficiency and accuracy of our proposed method.

\section{Example 1}

For a system described by [23]

$$
\begin{aligned}
& \dot{x}(t)=-x(t)+u(t)-0.5 u\left(t-\frac{2}{3}\right), \quad 0 \leq t \leq 1, \\
& x(0)=1.0, \\
& u(t)=0, \quad t \in\left[-\frac{2}{3}, 0\right]
\end{aligned}
$$

minimize

$$
J(X, U)=\frac{1}{2} \int_{0}^{1}\left\{x^{2}(t)+u^{2}(t)\right\} d t .
$$

Here, we solve this problem with hybrid functions. Note that in this example delay is applied on control only, and $\tau=\frac{2}{3}$. Suppose that

$$
x(t)=X^{T} H(t), \quad u(t)=U^{T} H(t), \quad x(0)=C_{0}^{T} H(t),
$$


Table $1 \mathrm{~J}$ values in Example 1 with $\mathrm{N}=3$

\begin{tabular}{ll}
\hline $\mathrm{M}$ & $\mathrm{J}$ value \\
\hline 3 & 0.195494339136585 \\
4 & 0.195494339136887 \\
5 & 0.195494339136887 \\
6 & 0.195494339136887
\end{tabular}

where $X^{T}, U^{T}, H(t)$ are defined previously and $C_{0}$ is the coefficient vector of 1 in term of hybrid functions expansion. If we integrate $\dot{x}(t)$ from 0 to $t$ and use (31)-(33)we have

$$
\int_{0}^{t} \dot{x}(s) d s=-\int_{0}^{t} x(s) d s+\int_{0}^{t} u(s) d s-\frac{1}{2} \int_{0}^{t} u\left(s-\frac{2}{3}\right) d s .
$$

For time delay control function one can easily find that

$$
\int_{0}^{t} u\left(s-\frac{2}{3}\right) d s= \begin{cases}0, & 0 \leq t \leq \frac{2}{3}, \\ U^{T} P_{h} D_{\tau_{2}} H(t), & \frac{2}{3} \leq t \leq 1 .\end{cases}
$$

So from (39) we obtain

$$
X^{T} H(t)-C_{0}^{T} H(t)=-X^{T} P_{h} H(t)+U^{T} P_{h} H(t)-\frac{1}{2} U^{T} D_{\tau_{2}} P_{h} H(t) .
$$

By deleting $H(t)$ from both sides and reordering of (41) we conclude that

$$
C^{*}=X^{T}-C_{0}^{T}+X^{T} P_{h}-U^{T} P_{h}+\frac{1}{2} U^{T} D_{\tau_{2}} P_{h}=0 .
$$

Now for cost functional $J$ we have

$$
\begin{aligned}
J & =\frac{1}{2} \int_{0}^{1}\left\{X^{T} H(t) H^{T}(t) X+U^{T} H(t) H^{T}(t) U\right\} d t \\
& =\frac{1}{2}\left[X^{T} L X+U^{T} L U\right],
\end{aligned}
$$

where $L$ is defined previously. Thus we have reduced the system as follows

$$
\begin{aligned}
\min & J & =\frac{1}{2}\left[X^{T} L X+U^{T} L U\right], \\
\text { s.to } & C^{*} & =X^{T}-C_{0}^{T}+X^{T} P_{h}-U^{T} P_{h}+\frac{1}{2} U^{T} D_{\tau_{2}} P_{h}=0 .
\end{aligned}
$$

We have solved this example with $N=3$ and $M=3,4,5,6$ by Maple 15 software with the CPU time of a core i5 in $2.45 \mathrm{~s}$. The values of $J$ are presented in Table 1 . The curves of state and control functions for $\mathrm{M}=6$ are shown in Fig. 1 .

\section{Example 2}

Consider the following delay optimal control system [14,19,24]

$$
\begin{aligned}
\min \quad J & =\frac{1}{2} \int_{0}^{2}\left\{x^{2}(t)+u^{2}(t)\right\} d t, \\
\text { s.t } \quad \dot{x}(t) & =x(t-1)+u(t), \quad 0 \leq t \leq 2, \\
x(t) & =1-1 \leq t \leq 0 .
\end{aligned}
$$




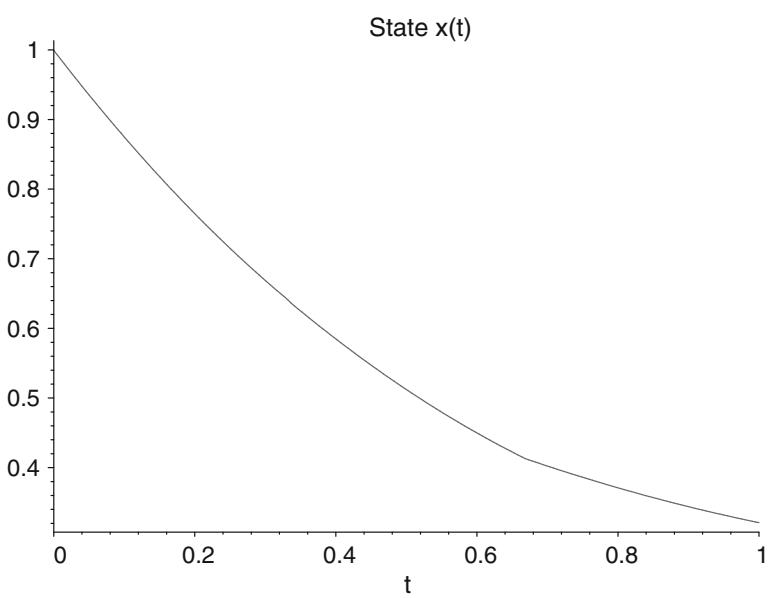

(a)

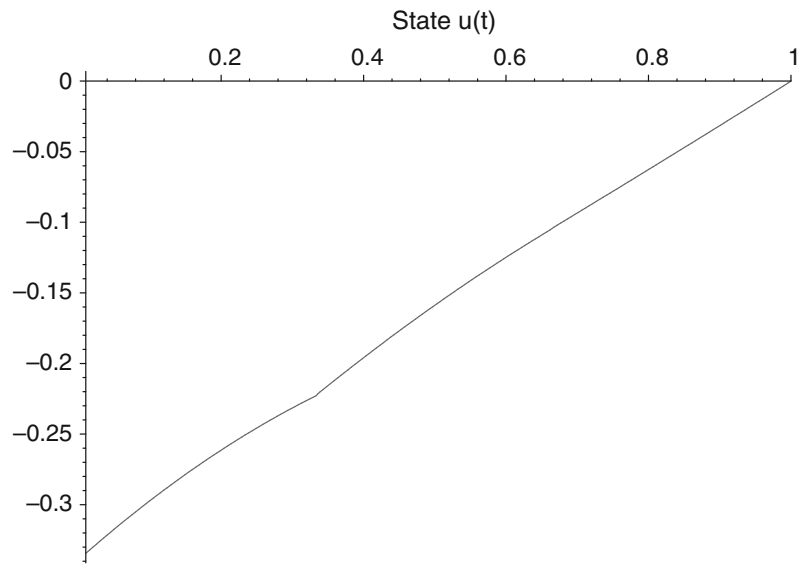

(b)

Fig. 1 State vector $x(t)$ and control $u(t)$ in Example 1. a State $x(t)$. b Control $u(t)$

Suppose that

$$
x(t)=X^{T} H(t), \quad u(t)=U^{T} H(t), \quad x(0)=C_{0}^{T} H(t) .
$$

We solve this example with $N=2$ and $M=4$. By expanding $x(0)$ in terms of hybrid functions we get

$$
x(0)=[1,0,0,0,1,0,0,0]^{T}=k_{1}^{T} H(t) .
$$

Also we have

$$
\int_{0}^{t} x(s-1) d s= \begin{cases}k_{2}^{T} H(t), & 0 \leq t \leq 1, \\ k_{3}^{T} H(t)+X^{T} D_{\tau} P_{h} H(t), & 1 \leq t \leq 2,\end{cases}
$$


Table 2 Results of Example 2 with $N=2$ and $M=6$

\begin{tabular}{|c|c|c|c|c|}
\hline \multirow[t]{2}{*}{ Time } & \multicolumn{2}{|l|}{ State $x(t)$} & \multicolumn{2}{|l|}{ Control u(t) } \\
\hline & Method of [23] & Peresent method & Method of [23] & Peresent method \\
\hline 0.00 & 1.0000 & 1.000000 & -1.9870 & -1.987936 \\
\hline 0.20 & 0.8364 & 0.836465 & -1.6566 & -1.657582 \\
\hline 0.41 & 0.7299 & 0.729501 & -1.3691 & -1.370340 \\
\hline 0.61 & 0.6794 & 0.678789 & -1.1143 & -1.143743 \\
\hline 0.81 & 0.6703 & 0.669425 & -0.9547 & -0.955914 \\
\hline 1.02 & 0.6971 & 0.695945 & -0.7947 & -0.795585 \\
\hline 1.22 & 0.7321 & 0.730322 & -0.6525 & -0.652911 \\
\hline 1.43 & 0.7716 & 0.770880 & -0.5031 & -0.495490 \\
\hline 1.63 & 0.8310 & 0.826640 & -0.3362 & -0.336116 \\
\hline 1.83 & 0.9163 & 0.910498 & -0.1631 & -0.162960 \\
\hline 2.00 & 1.0189 & 1.011921 & 0.0000 & 0.00000 \\
\hline
\end{tabular}

where

$$
\begin{aligned}
& k_{2}=\left[\frac{1}{2}, \frac{\sqrt{2} \sqrt{6}}{12}, 0,0,0,0,0,0\right]^{T}, \\
& k_{3}=[0,0,0,0,1,0,0,0]^{T},
\end{aligned}
$$

and $P_{h}$ is the operational matrix of integration given in (6) and $D_{\tau}$ is the delay operational matrix given by

$$
D_{\tau}=\left(\begin{array}{cc}
0 & I_{4} \\
0 & 0
\end{array}\right)
$$

where $I_{4}$ is 4-dimensional identity matrix. If we integrate (44) from 0 to $t$ and use (45)-(50) we have

$$
X^{T}-X^{T} D_{\tau} P_{h}-U^{T} P_{h}-k^{T}=0,
$$

where we have $k=k_{1}+k_{2}+k_{3}$.

The cost functional $J$ in (43) now changes to the following form

$$
J(X, U)=\frac{1}{2}\left(X^{T} L X+U^{T} L U\right) .
$$

Now we have reduced the system as follows

$$
\begin{aligned}
\min \quad J(X, U) & =\frac{1}{2}\left(X^{T} L X+U^{T} L U\right), \\
\text { s.t } \quad C^{*} & =X^{T}-X^{T} D_{\tau} P_{h}-U^{T} P_{h}-k^{T}=0 .
\end{aligned}
$$

The results obtained are given in Table 2. The results are compared well with the solutions obtained in [23].

Values of cost functional $J$ in [23], is reported as 1.6497 with $m=100$ and 1.6504 with $m=10$. Moreover in [14], $J$ value is reported as $2 \frac{2226}{2615}$. Approximate values of the cost function $\mathrm{J}$ with $N=2$ and for $M=4,5,6,8$ are given in the Table 3 . The curves of state and control functions for $\mathrm{M}=6$ are shown in Fig. 2 . 
Table 3 The cost functional $J$ in Example 2 with $\mathrm{N}=3$
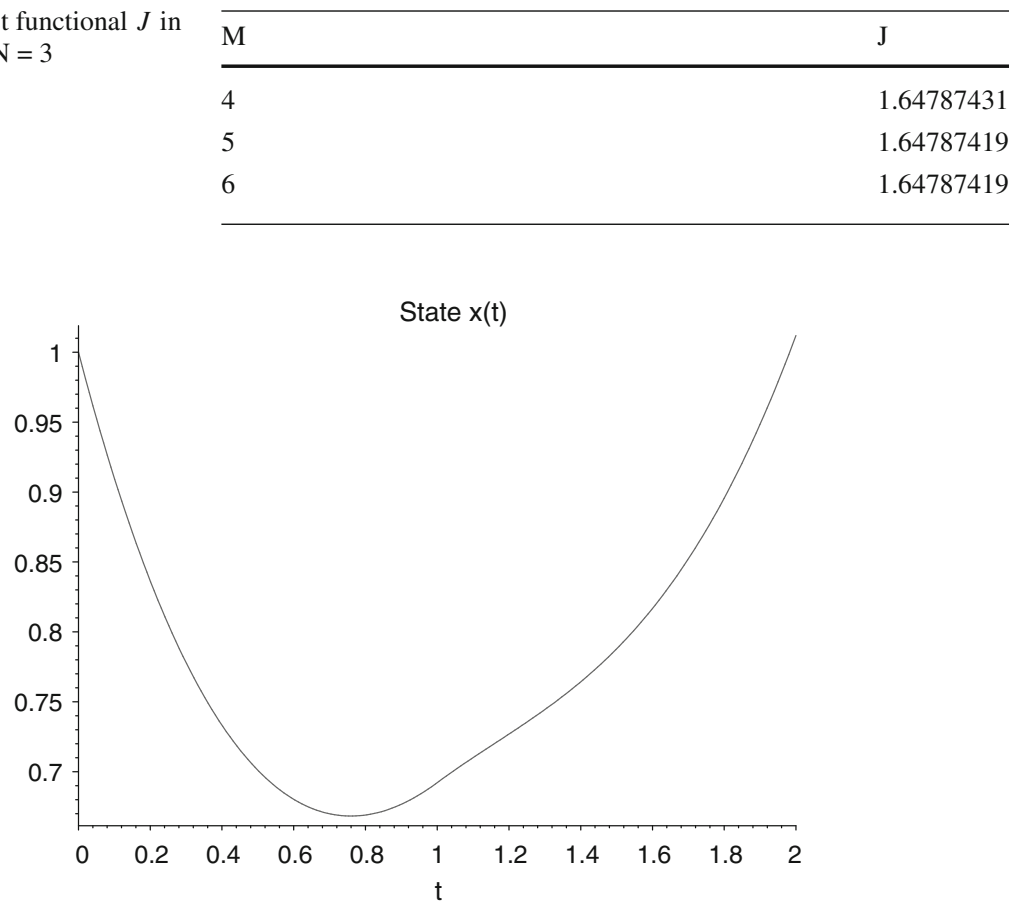

(a)

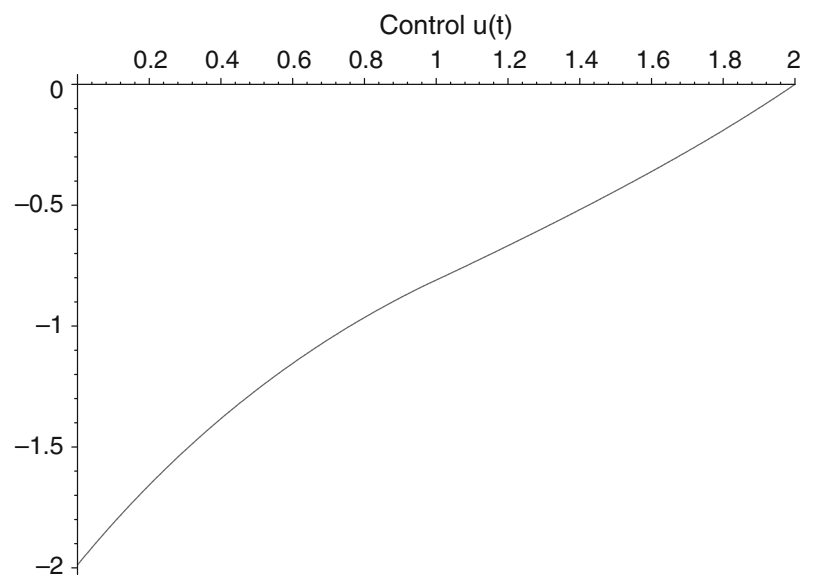

(b)

Fig. 2 State vector $x(t)$ and control $u(t)$ in Example 2. a State $x(t)$. b Control $u(t)$

\section{Example 3}

Consider the following delay optimal control system [5,9, 14,23]

$$
\min \quad J=\frac{1}{2} \int_{0}^{1}\left\{x^{2}(t)+\frac{1}{2} u^{2}(t)\right\} d t
$$




$$
\begin{aligned}
\text { s.t } \quad \dot{x}(t) & =-x(t)+x(t-1 / 3)+u(t)-\frac{1}{2} u(t-2 / 3), \quad 0 \leq t \leq 1, \\
x(t) & =1, \quad-1 \leq t \leq 0, \\
u(t) & =0, \quad-2 / 3 \leq t<0 .
\end{aligned}
$$

Here we have different delays in $\operatorname{state}\left(\tau_{1}=1 / 3\right)$ and $\operatorname{control}\left(\tau_{2}=2 / 3\right)$. The problem is to find the optimal control $u(t)$ which minimizes $\mathrm{J}$ in (53) subject to (54)-(56). The exact solution of this problem is not known, so we solve it by hybrid functions and by choosing $N=3$ and $M=4$. Suppose that

$$
x(t)=X^{T} H(t), \quad u(t)=U^{T} H(t), \quad x(0)=C_{0}^{T} H(t) .
$$

By expanding $x(0)$ in terms of hybrid functions we get

$$
x(0)=\left[\frac{\sqrt{3}}{3}, 0,0,0, \frac{\sqrt{3}}{3}, 0,0,0, \frac{\sqrt{3}}{3}, 0,0,0\right]^{T}=e_{1}^{T} H(t) .
$$

Also we have

$$
\begin{aligned}
& \int_{0}^{t} x\left(s-\frac{1}{3}\right) d s= \begin{cases}e_{2}^{T} H(t), & 0 \leq t \leq \frac{1}{3}, \\
e_{3}^{T} H(t)+X^{T} D_{\tau_{1}} P_{h} H(t), & \frac{1}{3} \leq t \leq 1,\end{cases} \\
& \int_{0}^{t} u\left(s-\frac{2}{3}\right) d s= \begin{cases}0, & 0 \leq t \leq \frac{2}{3}, \\
U^{T} D_{\tau_{2}} P_{h} H(t), & \frac{2}{3} \leq t \leq 1,\end{cases}
\end{aligned}
$$

where

$$
\begin{aligned}
& e_{2}=\left[\frac{\sqrt{3}}{18}, \frac{1}{18}, 0,0,0,0,0,0,0,0,0,0\right]^{T}, \\
& e_{3}=\left[0,0,0,0, \frac{\sqrt{3}}{9}, 0,0,0, \frac{\sqrt{3}}{9}, 0,0,0\right]^{T},
\end{aligned}
$$

and $P_{h}$ is the operational matrix of integration given in (6) and $D_{\tau_{1}}, D_{\tau_{2}}$ are the delay operational matrices given by

$$
D_{\tau_{1}}=\left(\begin{array}{ccc}
0 & I_{4} & 0 \\
0 & 0 & I_{4} \\
0 & 0 & 0
\end{array}\right)
$$

and

$$
D_{\tau_{2}}=\left(\begin{array}{ccc}
0 & 0 & I_{4} \\
0 & 0 & 0 \\
0 & 0 & 0
\end{array}\right)
$$

where $I_{4}$ is 4-dimensional identity matrix. By integrating (54) from 0 to $t$ and using (55)-(56) we have

$$
\begin{aligned}
\int_{0}^{t} \dot{x}(s) d s= & -\int_{0}^{t} x(s) d s+\int_{0}^{t} x\left(s-\frac{1}{3}\right) d s+\int_{0}^{t} u(s) d s-\frac{1}{2} \int_{0}^{t} u\left(s-\frac{2}{3}\right) d s, \\
X^{T} H(t)-e_{1}^{T} H(t)= & -X^{T} P_{h} H(t)+e_{2}^{T} H(t)+e_{3}^{T} H(t)+X^{T} D \tau_{1} P_{h} H(t) \\
& +U^{T} P_{h} H(t)-\frac{1}{2} U^{T} D_{\tau_{2}} P_{h} H(t) .
\end{aligned}
$$


Table 4 The cost functional $J$ in Example 3 with $\mathrm{N}=3$

\begin{tabular}{ll}
\hline BP method [9] & Peresent method \\
\hline $0.3723904(\mathrm{~N}=6)$ & $0.373112935(\mathrm{~N}=3, \mathrm{M}=4)$ \\
$0.3732373(\mathrm{~N}=9)$ & $0.373112935(\mathrm{~N}=3, \mathrm{M}=5)$ \\
$0.3731831(\mathrm{~N}=12)$ & $0.373112935(\mathrm{~N}=3, \mathrm{M}=6)$ \\
$0.3731359(\mathrm{~N}=21)$ & $0.373112935(\mathrm{~N}=3, \mathrm{M}=8)$ \\
$0.3731179(\mathrm{~N}=45)$ & $0.373112935(\mathrm{~N}=3, \mathrm{M}=10)$ \\
\hline
\end{tabular}

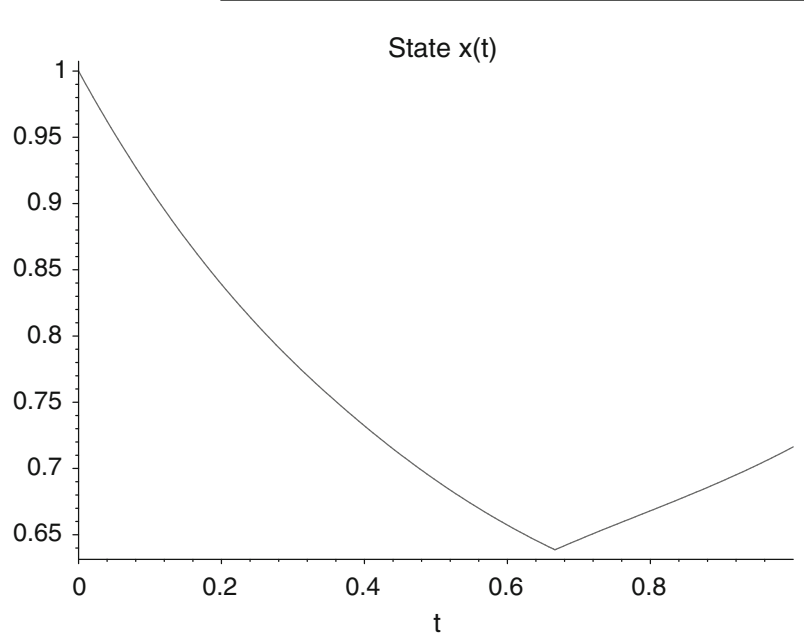

(a)

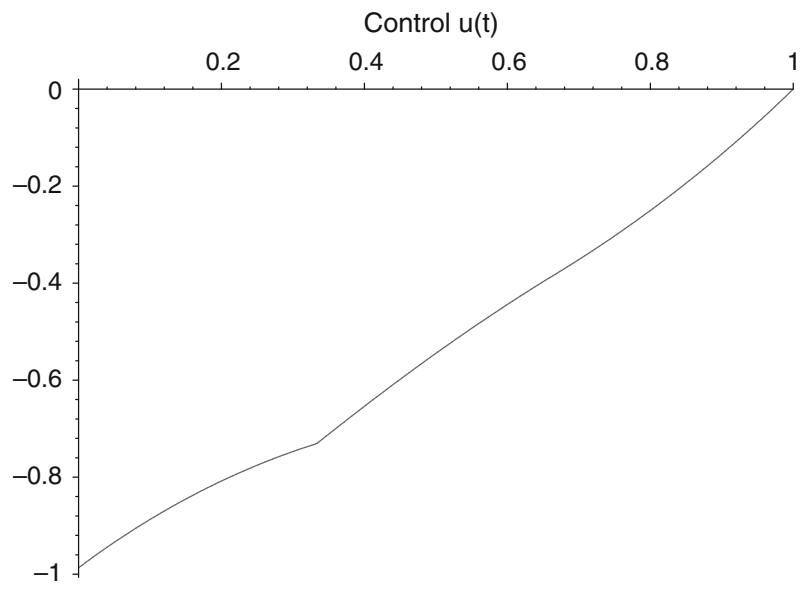

(b)

Fig. 3 State vector $x(t)$ and control $u(t)$ in Example 3. a State $x(t)$. b Control $u(t)$

So it is easily to find that

$$
X^{T}+X^{T} P_{h}-X^{T} D_{\tau_{1}} P_{h}-U^{T} P_{h}+\frac{1}{2} U^{T} D_{\tau_{2}} P_{h}-e^{T}=0,
$$

where we have $e=e_{1}+e_{2}+e_{3}$. 
The functional $J$ can be written as

$$
J(X, U)=\frac{1}{2}\left(X^{T} L X+\frac{1}{2} U^{T} L U\right),
$$

where $L$ is the dual operational matrix of $H(t)$. Thus the system is reduced as follows

$$
\begin{aligned}
\min \quad J(X, U) & =\frac{1}{2}\left(X^{T} L X+\frac{1}{2} U^{T} L U\right), \\
\text { s.t } \quad C^{*} & =X^{T}+X^{T} P_{h}-X^{T} D_{\tau_{1}} P_{h}-U^{T} P_{h}+\frac{1}{2} U^{T} D_{\tau_{2}} P_{h}-e^{T}=0 .
\end{aligned}
$$

Approximate values of the cost function $\mathrm{J}$ with $N=3$ and for $M=4,5,6,8,10$ are given in the Table 4 and are compared with the solutions obtained in [9]. The curves of state and control functions for $M=6$ are shown in Fig. 3 .

\section{Conclusion}

A new approach in solving optimal control of time delay systems with quadratic performance index has been proposed using hybrid of general block-pulse functions and orthonormal Taylor series. The operational matrices of integration, dual, product and delay are obtained and used to reduce the solution of optimal control problem to the solution of algebraic equations. The operational matrices of integration and product have many zeros and so they are sparse matrices which makes hybrid functions computationally very attractive. So the computational cost is decreased. Illustrative examples demonstrate that the method is valid.

\section{References}

1. Ge, S.S., Hong, F., Lee, T.H.: Adaptive neural network control of nonlinear systems with unknown time-delays. IEEE Trans. Autom. Control 48, 2004-2010 (2003)

2. Gollmann, L., Kern, D., Maurer, H.: Optimal control problems with delays in state and control variables subject to mixed control-state constraints. Optim. Control Appl. Methods 30, 341-365 (2009)

3. Gu, K., Kharitonov, V.L., Chen, J.: Stability of Time-Delay Systems. Birkhauser, Boston (2003)

4. Kharatishvili, G.L.: The maximum principle in the theory of optimal process with time-lags. Dokl. Akad. Nauk SSSR 136, 39-42 (1961)

5. Marzban, H.R., Shahsiah, M.: Solution of piecewise constant delay systems using hybrid of block-pulse and Chebyshev polynomials. Optim. Control Appl. Methods 32, 647-659 (2011)

6. Marcellan, F., Assche, W.V.: Orthogonal Polynomials and Special Functions (A Computation and Applications). Springer, Berlin (2006)

7. Lzaro, I., Anzurez, J., Roman, M.: Parameter estimation of linear systems based on walsh series. In: The Electronics, Robotics and Automotive Mechanics Conference, pp. 355-360. (2009)

8. Deb, A., Dasgupta, A., Sarkar, G.: A new set of orthogonal functions and its application to the analysis of dynamic systems. J. Frankl. Inst. 343, 1-26 (2006)

9. Mohan, B. M., Kar, S. K.: Optimal control of multi-delay systems via block-pulse functions. In: Fifth International Conference on Industrial and Information Systems (ICIIS), Mangalore, India, pp. 614-619. (2010)

10. Jiang, Z.H., Schaufelberger, W.: Block Pulse Functions and Their Applications in Control Systems. Springer, Berlin (1992)

11. Kung, F.C., Lee, H.: Solution and parameter estimation of linear time-invariant delay systems using Laguerre polynomial expansion. J. Dyn. Syst. Meas. Control 105, 297-301 (1983)

12. Khellat, F., Yousefi, S.A.: The linear Legendre wavelets operational matrix of integration and its application. J. Frankl. Inst. 343, 181-190 (2006)

13. Wang, X.T.: Numerical solutions of optimal control for time delay systems by hybrid of block-pulse functions and Legendre polynomials. Appl. Math. Comput. 184, 849-856 (2007) 
14. Wang, X.T.: Numerical solutions of optimal control for linear time-varying systems with delays via hybrid functions. J. Frankl. Inst. 344, 941-953 (2007)

15. Marzban, H.R., Razzaghi, M.: Solution of multi-delay systems using hybrid of block-pulse functions and Taylor series. J. Sound Vib. 292, 954-963 (2006)

16. Shaban, M., Kazem, S., Rad, J.A.: A modification of the homotopy analysis method based on Chebyshev operational matrices. Math. Comput. Model 57(5), 1227-1239 (2013)

17. Marzban, H.R., Razzaghi, M.: Optimal control of linear delay systems via hybrid of block-pulse and Legendre polynomials. J. Frankl. Inst. 341, 279-293 (2004)

18. Sezer, M., Dascioglu, A.A.: Taylor polynomial solutions of general linear differential-difference equations with variable coefficients. Appl. Math. Comput. 174, 1526-1538 (2006)

19. Farahi, M.H., Dadkhah, M.: Solving nonlinear time delay control systems by Fourier series. Int. J. Eng. Res. Appl. 4(6(Version 5)), 217-226 (2014)

20. Bernatz, R.A.: Fourier Series and Numerical Methods for Partial Differential Equations. Wiley, New York (2010)

21. Hoffman, K., Kunze, R.: Linear Algebra, 2nd edn. Prentice-Hall Inc, Englewood Cliffs (1971)

22. Kilicman, A., Al Zhour, Z.: Kronecker operational matrices for fractional calculus and some applications. Appl. Math. Comput. 187, 250-265 (2007)

23. Palanisamy, K.R., Rao, G.P.: Optimal control of linear systems with delays in state and control via Walsh function. Proc. IEE 130, 300-312 (1983)

24. Haddadi, N., Ordokhani, Y., Razzaghi, M.: Optimal control of delay systems by using a hybrid functions approximation. J. Optim. Theory Appl. 153, 338-356 (2011) 